\title{
Estudio de caso: Diseño de viviendas ambientales de bajo costo, Cuenca (Ecuador)
}

\author{
Antonio E. Barragán ${ }^{1,2}$, Pablo E. Ochoa ${ }^{3}$ \\ ${ }^{1}$ Carrera de Ingeniería Ambiental, Universidad Politécnica Salesiana, Cuenca, Ecuador. \\ ${ }^{2}$ Unidad Académica de Ingeniería, Facultad de Ingeniería Eléctrica, Universidad Católica de Cuenca, \\ Cuenca, Ecuador. \\ ${ }^{3}$ Ochoa y Contreras Arquitectos, Cuenca, Ecuador.
}

Autor para correspondencia: tono_barragan@yahoo.es

Fecha de recepción: 10 de julio 2013 - Fecha de aceptación: 20 de enero 2014

\section{RESUMEN}

Una vivienda ecológica tiene como principal objetivo minimizar el impacto ambiental, utilizando tanto como sea posible materiales locales, así como reduciendo el consumo de agua y energía. Este artículo presenta el diseño de una vivienda ecológicamente sustentable de bajo costo que garantiza la calidad de vida de sus residentes. Como resultado se tiene una vivienda modular que se puede construir en un terreno de $81 \mathrm{~m}^{2}$, y está diseñada bajo condiciones del clima local. Se utilizó el software de simulación ECOTECT 3D, para validar el diseño. El análisis revela que es factible construir en la ciudad de Cuenca, Ecuador, viviendas sociales con características bioclimáticas básicas, bajo condiciones económicas y ambientales particulares. El enfoque planteado puede ser fácilmente extrapolable a otras locaciones utilizando parámetros regionales de clima, así como los materiales locales disponibles.

Palabras clave: Viviendas, viviendas ecológicas, vivienda modular de bajo costo, uso eficiente de agua, energía y de materiales.

\begin{abstract}
The main objective of green homes is to minimize the overall environmental impact by using as much as possible local materials and reducing the water and energy consumption, both during the construction and the lifetime of the home. This paper presents the design of a low cost sustainable home guaranteeing the quality of life of the residents. The result is a modular house with a total habitable (built-up) area of $81 \mathrm{~m}^{2}$, taking optimal advantage of local climate. The Autodesk ECOTECT 3D simulation software was used to validate the concept. The analysis revealed that it is feasible to build in the city of Cuenca, Ecuador, social homes meeting the basic bioclimatic requirements given the local economic and environmental conditions. The approach is easy extrapolatable to other locations using the regional climate parameters and local available building materials.
\end{abstract}

Keywords: Homes, green homes, passive design, modular low cost home, efficient use of water, energy and materials.

\section{INTRODUCCIÓN}

En los países en vías de desarrollo se experimenta un crecimiento demográfico, que está causando presión en la infraestructura existente, que afecta al transporte, vialidad, calidad del agua, recolección de desechos y a la salud pública (Cerón y col., 2013). Las ciudades ecuatorianas no se escapan de esta tendencia, pues tienen un alto índice de crecimiento, que se extiende en unos casos a través de 
invasiones en sectores próximos a las grandes ciudades o en zonas críticas e inestables (Paz Jácome y Ulloa López, 2006).

Para permitir dimensionar la situación de habitabilidad se suelen utilizar varios indicadores que capturan distintos matices del problema habitacional: infraestructura, servicios básicos, calidad de la vivienda y la densidad ocupacional. Considerando esto, Larrea (2010) determinó que el déficit habitacional en el Ecuador, desde la década de los ochenta ha disminuido de un $83 \%$ a un de $64 \%$, en la actualidad. A pesar de esta notable mejora, se distingue que en el área urbana la mitad de las viviendas son adecuadas, es decir: tienen agua, alcantarillado, electricidad de la red pública, recolección de basura, paredes y pisos adecuados, servicio higiénico exclusivo y menos de tres personas por cuarto.

La disminución del déficit de vivienda, por tanto, se traduce en la posibilitad de construir viviendas que reúnan criterios de habitabilidad, o en otras palabras, se debe construir viviendas que reúnan características adecuadas de confort. Según el Instituto Nacional de Estadísticas y Censos (INEC), el déficit habitacional en el Ecuador es de más 1,4 millones de viviendas, y se estima que se necesitan más de 50.000 viviendas al año para cubrir el crecimiento poblacional $(1,2 \%)$ y 150.000 para superar el déficit cualitativo, relacionado con la calidad de la infraestructura. En el caso particular de la ciudad de Cuenca, el déficit de vivienda en el cantón es de 45.000 unidades.

$\mathrm{Al}$ igual que sucede en México o Brasil, en el Ecuador el crecimiento urbano, ha incrementado la demanda en energía eléctrica, así como la energía utilizada para el transporte, industria y residencia (Cerón y col., 2013; Bodach, 2010). En las últimas 4 décadas Ecuador, se ha vuelto un país exportador de energía; sin embargo, sigue siendo un país que no tiene autoabastecimiento energético. Tanto en el sector eléctrico como en los hidrocarburos, ha sido evidente, la falta de inversión para mantener capacidad instalada, ya sea por generación eléctrica o en su capacidad de procesar derivados del petróleo. En el caso particular del sector residencial, se produjo una reducción anual de la intensidad energética promedio del 2,3\%, provocada en parte por el cambio del estilo de vida, preferencias de consumo, el cambio en el uso de energía, leña por gas licuado de petróleo o por la penetración de la electricidad. Esto junto con el proceso de industrialización y el incremento de transporte, provocó que para el año 2006, el consumo energético en el sector residencial sea del 19\%, en contraste con el $28 \%$ que representaba el consumo en 1980. De lo anterior, se advierte la necesidad de dotar a la población de viviendas dignas, que garanticen el confort, y que disminuyan la presión sobre el incremento constante de energía a nivel residencial, en otras palabras para el futuro se requieren viviendas "más accesibles, más sostenibles" (Seyfang, 2010).

El déficit habitacional que existe en el país se debe a la falta de soluciones habitacionales de bajo costo económico. El "bajo costo" de una vivienda, suele ser sinónimo de mala calidad, es decir, de un producto que no reúne las características de habitabilidad adecuadas. Fusionar, el aspecto económico, con las nuevas exigencias establecidas por varias organizaciones que han desarrollado códigos y normas para que los gobiernos o profesionales promuevan el desarrollo de proyectos que reúnan criterios de diseño ecológico, es el reto futuro (GhaffarianHoseini y col., 2013). En Assefa y col. (2010), se destacan varios métodos, entre los que se incluye BREEAM (Environmental Assessment Method, Reino Unido), CASBEE (Comprehensive Assessment System for Built Environment Efficiency, Japon), o la LEED (Leadership in Energy \& Environmental Design, Estados Unidos).

A pesar de que no se dispone de códigos técnicos ecuatorianos que reúnan los principios del diseño ecológico, esta investigación recoge los principios resumidos en Abdel y Aboulgheit (2012), para proponer estrategias de diseño arquitectónico que no solo consideren los fundamentos de una eco vivienda, sino también sean viviendas de carácter social. No solo se busca, por tanto, proponer un diseño de vivienda, sino se busca establecer una serie de métodos y herramientas de diseño para que los futuros proyectistas arquitectónicos tengan una hoja de ruta en la panificación de proyectos sostenibles locales (GhaffarianHoseini y col., 2013). La concepción de un diseño que reúna principios bioclimáticos, de bajo costo, fomentará y motivará a que las estrategias sean utilizadas, ya que la falta de entendimiento de estos principios, es una de las barreras para la aplicación masiva en el desarrollo de proyectos habitacionales (Zhang y col., 2011). Para delimitar el análisis, se ha establecido como zona de estudio al cantón Cuenca, que concentra $88,9 \%$ de población de la provincia del Azuay. Según datos estadísticos de la Empresa Municipal de Vivienda de la ciudad de Cuenca, en el 2011 el déficit 
habitacional en ésta fue de entre 35.000 a 45.000 soluciones habitacionales. La hipótesis del estudio es que es posible diseñar una vivienda social con criterios bioclimáticos en esta localidad.

A nivel local, el uso de las estrategias de diseño que posibiliten incluir criterios de arquitectura verde, ayudará a que estas prácticas sean aceptadas en el mercado inmobiliario, pues, a mediano o largo plazo, permitirán mejorar el desempeño de la edificación, disminuir los costos de operación, mejorar las condiciones de salud de los habitantes y promoverá el ahorro de energía (Zhang y col., 2011). Paralelamente, se concibe la propuesta como parte de una innovación o "el uso de nuevas ideas", que en este caso entrelazan a la tecnología y al cambio social (Seyfang, 2010).

\section{LA CONCEPCIÓN DE VIVIENDAS AMBIENTALES DE BAJO COSTO}

La concepción de una vivienda, tradicionalmente ha sido ideada sin tomar en cuenta el entorno local; sin embargo, la elección adecuada de materiales, el uso de energía, el ambiente interior y exterior son aspectos de importancia que valen la pena considerar (Assefa y col., 2010). Se propone diseñar viviendas sociales, modulares y progresivas, con atributos diferentes a los usuales, que permitan la construcción prefabricada y montaje en seco, con materiales y sistemas constructivos apropiados para el entorno natural local, que a su vez admitan versatilidad, rapidez y posibilidades de autoconstrucción. Todo esto bajo el concepto de una vivienda de carácter social. En este sentido y asumiendo lo establecido en Assefa y col. (2010), GhaffarianHoseini y col. (2013), Seyfang (2010) y Wekesa (2010) la vivienda se concibe de forma que integre las dimensiones de sostenibilidad: ambiente (viviendas que aprovechen las condiciones ambientales, el uso de fuentes renovables de energía); económico (viviendas con bajos costos de inversión) y social (dar soluciones habitacionales que garanticen la calidad de vida de sus ocupantes).

El concepto de una eco-vivienda tiene sus orígenes a principios de los setenta, y considera la necesidad de crear viviendas, que respeten la salud de las personas y del planeta. Esta concepción nace de la aceptación de que la energía y materiales provienen de fuentes finitas, y de la necesidad de mantener niveles bajos de contaminación (Seyfang, 2010). En los ochenta aparece el concepto de arquitectura verde, que reúne criterios de conservación de energía, clima, mínimo uso de recursos y respeto a los usuarios, es decir se concibe una concepción holística (Malmqvist y Glaumann, 2009). En la actualidad, se admite que una vivienda sostenible, es aquella que consume pocos recursos, produce menos desperdicios, se adapta al clima local y asegura que las decisiones actuales no repercutan en las futuras generaciones. El diseño de un ambiente confortable y eficiente que reúna la anterior definición, considera varios aspectos: una integración sostenible y amigable con el ambiente y el uso de materiales de bajo impacto ambiental (Assefa y col., 2010).

Puesto que el manejo eficiente de energía es uno de los objetivos del eco diseño (Peuportier $y$ col., 2013), un diseño sostenible permitirá a los residentes reducir la polución a través de la selección de materiales con bajas emisiones, así como el acceso a la luz solar y el control óptimo de la luz para garantizar el confort (Assefa y col., 2010). Estos dos aspectos, el manejo de la energía y calidad del ambiente interior, son precisamente los más significativos impactos ambientales relacionados a las viviendas (Malmqvist y Glaumann, 2009). En cuanto al ambiente interior, la vivienda debe concebir condiciones adecuadas de calidad de vida y salud. La mala calidad de aire, niveles de iluminación, altas emisiones de gases y efluentes o materiales adversos pueden afectar a los ocupantes (Assefa $y$ col., 2010).

El diseño de una vivienda de interés social no tiene por qué alejarse de estas características, pudiendo acoger los principios del diseño sostenible, tales como: eficiencia en el diseño, eficiencia energética, uso eficiente de agua, uso de materiales eficientes, calidad ambiental interior, óptimo mantenimiento y operación, reducción de desechos comunes y tóxicos (Abdel y Aboulgheit, 2012; Assefa y col., 2010):

- Un diseño eficiente, repercute en los costos, en el rendimiento de la edificación y en la disminución del uso de materiales (Cabeza y col., 2013). Este último tópico tiene importancia 
pues a nivel mundial la construcción de edificios y obras civiles consume el 60\% de la materia prima que se extrae de la litósfera (Zabalza y col., 2011).

- El manejo eficiente del uso de energía en el transcurso de la vida útil de la vivienda, se logra a partir de un diseño que evite fugas de energía y niveles adecuados de iluminación. Así mismo la orientación de las ventanas y paredes, además de la colocación de cubiertas, árboles y el manejo adecuado de sombras, condicionarán el confort tanto en invierno como en verano. En el mismo sentido una colocación adecuada de las ventanas, provee mayor cantidad de luz natural y por tanto disminuye el consumo de energía en procesos como la calefacción, luz artificial u otras aplicaciones (Cerón y col., 2013). El uso de energías renovables, especialmente la solar térmica para el calentamiento de agua permite una reducción significativa de costos de energía (GhaffarianHoseini y col., 2013).

- El uso eficiente de agua reduce su consumo, además permite la reutilización, ya sea utilizando inodoros de bajo consumo, o el uso del agua de lluvia para determinadas actividades como riego. De hecho, en las áreas urbanas el uso de agua lluvia es considerada una estrategia para hacer frente a la escasez y degradación del agua (Ramón, y col, 2011).

- En cuanto al uso de materiales eficientes, el interés es utilizar por ejemplo madera de bosques con sello verde, rápidamente renovable, o productos que no sean tóxicos, reusables o reciclables. Otra vía puede ser el uso de materiales cuya fabricación y obtención estén cerca de donde se emplazará las viviendas (Seyfang, 2010; Zander y Sandström, 2012; Cabeza y col., 2013). Aunque, esto último, es un tema a discutir pues se considera que los impactos ambientales relacionados con el transporte son bajos, si se compara con los producidos por la operación y la construcción de la vivienda (Peuportier y col., 2013; Pacheco-Torga y Jalali, 2012). Sin embargo, en Cabeza y col. (2013), se anota que la producción de materiales que están fuera del sitio de construcción, representan el 75\% del total de la energía utilizada en la construcción. Por otro lado, el uso de materiales que pueden resultar baratos a la larga podrían representar altos costos energéticos o de mantenimiento, mientras que materiales más elaborados podrían ser más eficientes en el tiempo de vida de la edificación (Zabalza y col., 2011).

- La mejora de la calidad ambiental interior (IEQ o Indoor Environment Quality Enhancement), busca proveer confort a los ocupantes de la vivienda. El diseño de la vivienda nuevamente es indispensable para lograr esto, buscándose una adecuada calidad de aire, condiciones térmicas y lumínicas óptimas (Assefa y col., 2010).

- Una óptima operación y mantenimiento permitirán que la vivienda se mantenga adecuadamente en el tiempo.

El diseño de la vivienda que englobe los anteriores conceptos, deberá considerar la dimensión de sostenibilidad: economía, ambiente y sociedad. Esto se enmarca en las características de una vivienda social, que aproveche las condiciones ambientales para garantizar el confort ambiental interno. En la Fig. 1, se esquematiza los elementos que caracterizan el diseño, y se indica que la propuesta parte del análisis del entorno natural y climático, así como de las condiciones económicas que definen a una vivienda de carácter social; además, en el diseño se establecen condiciones de confort ambiental, necesarias para que el ser humano desarrolle sus actividades diarias (Edwards, 2013).

Las condiciones ambientales se examinan utilizando las siguientes herramientas bioclimáticas: el diagrama psicosométrico, el diagrama de variación de temperatura, la geometría solar, mapa estereográfico y ángulos solares. Para determinar las condiciones de una vivienda considerada como social, se realizó un análisis a varios planes de vivienda denominados como "popular", además de la normativa local que delimita las áreas mínimas de construcción. La información recolectada permitió definir una Matriz de Valoración, que recoge una serie de elementos que caracterizan este tipo de viviendas en la zona de estudio, así como ayudan a la programación arquitectónica. Como parte del diseño se establecieron criterios que recogen elementos de la arquitectura bioclimática, como el reciclaje de aguas, elección de materiales, o espacios para un uso futuro de energías renovables, particularmente la solar térmica. 
Por otro lado, el uso de un software amigable, fue esencial, pues además de permitir la presentación en 3D del proyecto, facilita la cuantificación de los volúmenes de material a ser usados, su tipo, así como el desempeño lumínico y térmico de la vivienda (Peuportier y col., 2013). El software elegido, fue el Autodesk ECOTECT, el cual facilita la administración de los espacios, según el criterio del diseñador, calcula los valores de temperatura $\left({ }^{\circ} \mathrm{C}\right)$ y luminosidad (lux), en definitiva, valida que las condiciones requeridas sean las adecuadas.

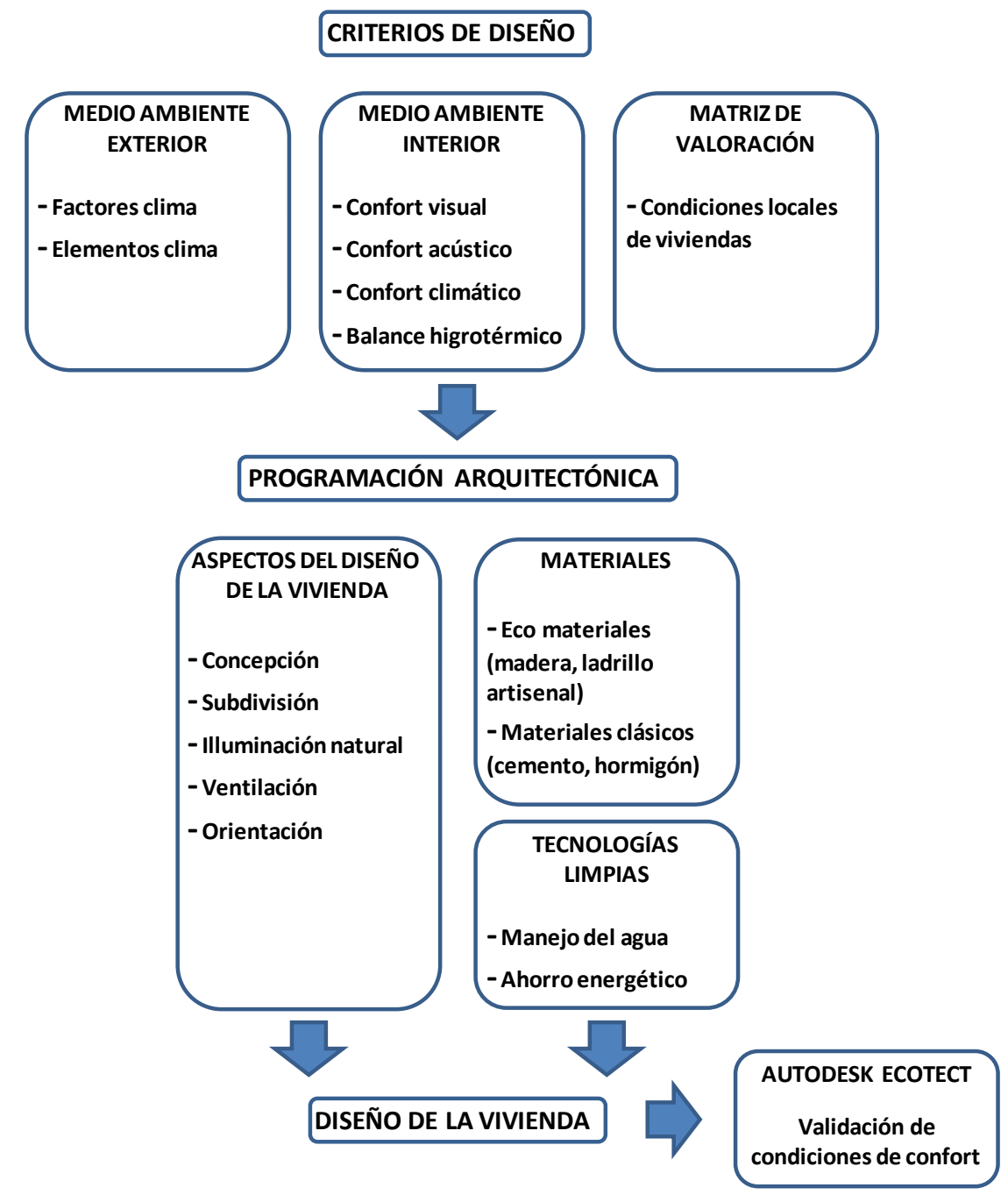

Figura 1. Fases del diseño de una vivienda social con criterios bioclimáticos.

\section{CRITERIOS DE DISEÑO}

\subsection{Medio ambiente exterior}

Para un diseño pasivo de una vivienda que esté adaptada de forma óptima a las condiciones climáticas locales, se requiere esencialmente dos tipos de información: los factores clima y los elementos clima. Los factores clima son el conjunto de circunstancias geográficas que influyen en los elementos clima, como: (i) la latitud y longitud, y (ii) la altitud. Entre los elementos clima considerados, se tiene: (i) temperatura y humedad, (ii) precipitación, y (iii) nubosidad y heliofanía. Está claro que el uso de un alto número de variables hará que el proyecto se vuelva más complejo; en este sentido la experiencia del diseñador será significativa (Assefa y col., 2010) para determinar la importancia de uno u otro dato. A continuación se presenta una descripción de los factores del clima en el área de estudio, 
seguido por un resumen de los elementos del clima. La caracterización es basada sobre datos diarios monitoreados durante un periodo de 5 años en la estación meteorológica del Centro de Estudios Ambientales de la Universidad de Cuenca (datos de temperatura y humedad), y de la estación climatológica del aeropuerto Mariscal Lamar (para el resto de elementos clima).

- Los factores clima, determinan la orientación y estructura de la edificación (latitud y longitud), o las condiciones de temperatura y clima (latitud). Así la ciudad de Cuenca, ubicada en un valle interandino de la sierra sur ecuatoriana, a una latitud de $2^{\circ} 53^{\prime} 12^{\prime \prime} \mathrm{S}$, longitud $79^{\circ} 09^{\prime} \mathrm{W}$ y una altitud de $2550 \mathrm{msnm}$, presenta una climatología típica interandina.

- Temperatura $\left[{ }^{\circ} \mathrm{C}\right]$ : La provincia del Azuay, en donde se encuentra la ciudad de Cuenca, está ubicada en la zona montañosa sur de la cordillera de los Andes, y según la escala de Köppen tiene un clima Mesotérmico semi-húmedo. La temperatura media anual de Cuenca y sus alrededores oscila alrededor de 15 a $16^{\circ} \mathrm{C}$. Entre los meses de octubre a febrero la temperatura promedio está más próxima a los niveles del confort, mientras que los meses más fríos se los identifica entre junio y septiembre.

- Humedad relativa [\%]: La humedad relativa es irregular; sin embargo, tiene mayor porcentaje entre el periodo de marzo y mayo y menor humedad en los demás meses. Es de notar que su variación no es significativa $(\leq 10 \%)$.

- Precipitación [mm - días lluvia]: Las precipitaciones se distribuyen en una estación lluviosa de octubre a mayo y una estación seca de junio a septiembre. Los meses de mayores precipitaciones se dan en dos periodos, el primero entre marzo y abril siendo la temporada de mayores precipitaciones y un segundo periodo en los meses de octubre y noviembre en menor medida. Los meses más secos se identifican entre julio y agosto. En cuanto a los valores de "días lluvia" se tienen, los picos más altos entre febrero y abril, llegando a bordear el $75 \%$ del mes con descargas, mientras que el promedio anual es de 50\%. Al disponer de las cantidades de lluvia mensuales sobre las cubiertas del proyecto se podrá calcular y dimensionar el sistema de reciclaje de agua lluvia para usos secundarios como limpieza, riego o sanitarios.

- Heliofanía [horas sol]: Los meses de mayor radiación solar directa dentro de la zona de estudio se encuentran en julio y agosto con valores de 186 horas de sol por mes, que representan un poco más del $50 \%$ de radiación directa.

- Nubosidad [octas]: Según los datos de la estación meteorológica, los meses de febrero hasta mayo, agosto, octubre y noviembre, presentan los mayores valores de nubosidad, 7/8. Esta información permite determinar la presencia de radiación difusa, esencial para el dimensionamiento de sistemas solares térmicos.

En cuanto a la evaluación climática, también llamada confort medioambiental, se analiza en forma individual las cartas climáticas de la zona de estudio, tal como el diagrama psicosométrico, el diagrama de valoración diaria de temperatura y la geometría solar.

- El Diagrama psicosométrico, es un ábaco en el que se manifiestan múltiples parámetros ambientales medibles en escala gráfica como temperatura, humedad absoluta, humedad relativa, punto de rocío, entalpía, entre otros. Este gráfico describe las estrategias arquitectónicas bioclimáticas y las necesidades de equilibrio térmico para los espacios interiores. El diagrama permite, con los valores máximos de temperatura y humedad, determinar la estrategia bioclimática más adecuada para el proyecto (Murillo, 2011). Utilizando el diagrama, se estableció que el diseño de la vivienda debe considerar sistemas solares pasivos, es decir: ganancias térmicas por radiación solar directa. Esto debido a que las temperaturas ambiente están por debajo de los niveles de confort climático, y la radiación es el principal regulador del clima de los ambientes. Los sistemas solares pasivos en este caso, consisten principalmente en definir la orientación con respecto al recorrido solar, con el fin de incrementar las ganancias térmicas para el interior de la vivienda.

- El Diagrama de variación diaria de temperatura, permite determinar con alto nivel de precisión los valores de temperatura a cada hora, para el día promedio de cada mes. Con los datos meteorológicos locales, se ubican las horas del día en donde no se dispone de 
temperaturas de confort. Luego se establecen estrategias de compensación térmica para regular el clima interior. Con el uso de este diagrama, se determinó que a las 6 h00 se tiene la mínima temperatura y a las 14 h00 la máxima. La información recolectada indicó que la variación diaria de las temperaturas de confort climático está entre $18^{\circ} \mathrm{C}$ y $24^{\circ} \mathrm{C}$. Además, para cada mes se manifiestan a partir de las $12 \mathrm{~h} 00$ hasta las $17 \mathrm{~h} 00$, lo que representa aproximadamente el $25 \%$ del total del día, teniendo el restante $75 \%$ temperaturas por debajo de los niveles requeridos. Estos datos también permiten justificar la necesidad de orientar la edificación de forma que se optimice las ganancias de temperatura.

- La Geometría solar, proporciona la posición del sol a cada hora del día en cualquier latitud y altitud del planeta. Estos datos permiten establecer estrategias para captar la energía solar, o en su defecto, definir estrategias de protección (Neila, 2001). Con la carta solar, además se determinan las condiciones ambientales del lugar a diferentes horas del día, para luego enfrentarlas a datos de flujos y ocupación, con el fin de proyectar, condiciones de iluminación, temperatura y radiación en cada uno de los espacios de la estancia. Asimismo, se puede conocer la hora y la época del año en la que una edificación provocará sombra a otras edificaciones vecinas. Con la carta solar, se puede conocer los ángulos solares de azimut y altura $^{1}$ y estudiarlos en un modelo 3D, para de esta manera trazar mapas de radiación y sombra, sobre los objetos a estudiar. Este gráfico se ubica sobre el objeto en estudio y entrega datos específicos de ubicación y de incidencia de la radiación, con el fin de diseñar las estrategias necesarias para el acondicionamiento climático natural. Se llega a establecer un conjunto de ángulos solares para la ubicación geográfica de la zona de estudio, con una variación de 30 minutos durante las horas de sol, para los doce meses del año. Para el diseño se determinó el Mapa Estereográfico para la ubicación geográfica de la Ciudad de Cuenca ${ }^{2}$.

\subsection{Medio ambiente interior}

Con el fin de establecer el confort medioambiental, se establecen cuatro condiciones:

- Confort visual: La comodidad visual depende de un sentido informativo, "de la capacidad de ver con el mínimo esfuerzo aquello que el ser humano quiere observar" (Serra, 2002), o en otras palabras, está relacionado con la cantidad adecuada de iluminación y su distribución de forma que se pueda realizar una determinada actividad (70 lux en pasillos a 500 lux en dormitorios).

- Confort acústico: Permite definir los niveles de ruido aceptable, y persistencia de un sonido en un local (45 dBA a $50 \mathrm{dBA}$, en áreas residenciales) (Gonzalez, 2004).

- Confort climático: El confort climático está relacionado con la calidad del aire para la respiración, así como su renovación en un local, y con confort térmico, donde intervienen los complejos fenómenos energéticos de intercambio de energía entre el cuerpo y el ambiente $\left(18^{\circ} \mathrm{C}\right.$ a $\left.24^{\circ} \mathrm{C}\right)($ Serra, 2002).

- Balance higrotérmico: Está relacionado con la pérdida de calor del cuerpo humano; una mayor velocidad implica sensación de frío; y una menor velocidad, sensación de calor (40\% a 60\%).

El parámetro fundamental es la temperatura, pero no se puede olvidar el contenido de humedad en el ambiente, el movimiento del aire, la actividad y el tipo de arropamiento (Higueras, 2006).

Según Malmqvist y Glaumann (2009), la calidad de aire, el confort climático, el confort acústico y el visual, permiten definir la satisfacción de los usuarios de una vivienda. El proyecto, analiza, tanto el confort visual como el confort climático, ya que el confort acústico es imposible de calcular si no se tiene una ubicación fija y una fuente de sonido específica.

\footnotetext{
${ }^{1}$ Son ángulos solares. Azimut: Angulo de posición solar medido en el plano horizontal desde el norte en dirección de las manecillas del reloj. Altura: Angulo de posición solar medido en el plano vertical.

${ }^{2}$ University of Oregon: http://solardat.uoregon.edu/PolarSunChartProgram.php.
} 


\subsection{Matriz de valoración}

Con el fin de marcar determinantes de diseño se han analizado varios planes de vivienda recientes o que se encuentran en proceso de construcción que promueven entidades públicas o privadas con el fin de crear una "Matriz de Valoración" que permita precisar índices medibles de las condiciones espaciales, arquitectónicas y económicas. La evaluación de cada uno de estos indicadores se las realizó sobre los siguientes planes de vivienda: "Racar II" de Mutualista Azuay; "Por Fin Casa - Rio Amarillo" de Por Fin Casa; "Molinos de Capulispamba" de EMUVI; "Rieles de Monay" de EMUVI; "Jardines del Valle" de Multicasa; "Casa Lista" de Mutualista Pichincha; "Casa Tipo MIDUVI" de MIDUVI; y Proyecto Habitar 5.000 de "Constru Hábitat".

En la matriz de valoración se recolectó variada información que estableció las características de los proyectos habitacionales (ambientes, sistemas constructivos, áreas, vanos, normativas, densidad ocupacional, costos o posibilidad de crecimiento). Todo esto con el fin de definir particularidades de las viviendas, que los usuarios están dispuestos a adquirir, así como también las características de las urbanizaciones a las que pertenecen. Los ítems de análisis de la vivienda se los describe a continuación, y en la Tabla 1, se anotan rangos de la información obtenida:

a) Urbanización: describe los metros cuadrados y sus relaciones en porcentajes de lotes, vías, veredas, parqueaderos, áreas verdes, equipamientos y otros espacios que dispone cada conjunto. Los planes se han desarrollado en terrenos rústicos planificados como zonas de expansión rural y no cuentan con áreas de parqueo ni plazas previstas para expansión, el equipamiento es escaso y no disponen de índices adecuados de áreas verdes.

b) Normativa: detalle de número de pisos, retiros y densidad ocupacional. Las viviendas no superan los dos niveles y la disposición de espacios son el resultado del diseño. El valor promedio del terreno es de alrededor de 15.000 USD, mientras que de las viviendas es de 22.000 USD.

c) Vivienda: cantidad de metros cuadrados de planta baja, planta alta y vivienda total.

d) Usuarios: número de habitantes por vivienda y metros cuadrados por terreno y vivienda.

e) Costos: precios de terreno, vivienda, y el costo por usuario y por metro cuadrado.

f) Inversión: forma de pago y avance de ventas del promotor. El financiamiento de las viviendas es promovido por bancos privados, Bono de la Vivienda y/o préstamos del BIESS (Banco Ecuatoriano del Instituto de Seguridad Social).

g) Crecimiento: capacidad o no de la vivienda de ampliarse sin afectar la estructura y estética. Las viviendas estudiadas no consideran crecimiento progresivo.

h) Ambientes: permite conocer cuáles son los espacios mínimos que el mercado de bienes raíces demanda para el diseño de la vivienda. Las viviendas disponen de sala, comedor, cocina, dormitorios, y una cantidad de baños proporcional a la cantidad de dormitorios. Además, tienen otros servicios como patio, garaje y lavandería.

i) Sistemas constructivos: materiales predominantes y tipo de acabados constructivos que tiene la vivienda. Los ejemplos analizados muestran sistemas constructivos tradicionales, salvo particularidades en el uso de mampostería para muros portantes elaborados con formaletas y paneles de prefabricados para pisos y losas.

j) Vanos/lleno: permite conocer la orientación y proporción de las ventanas sobre el muro de cada fachada para conocer el tipo y cantidad de iluminación natural con la que se dispone. Ninguno de los planes de vivienda ha considerado la orientación de sus fachadas como estrategia de diseño bioclimático. 
MASKANA, Vol. 5, No. 1, 2014

Tabla 1. Elementos y rangos de valor de la Matriz de Valoración de proyectos de vivienda de carácter social.

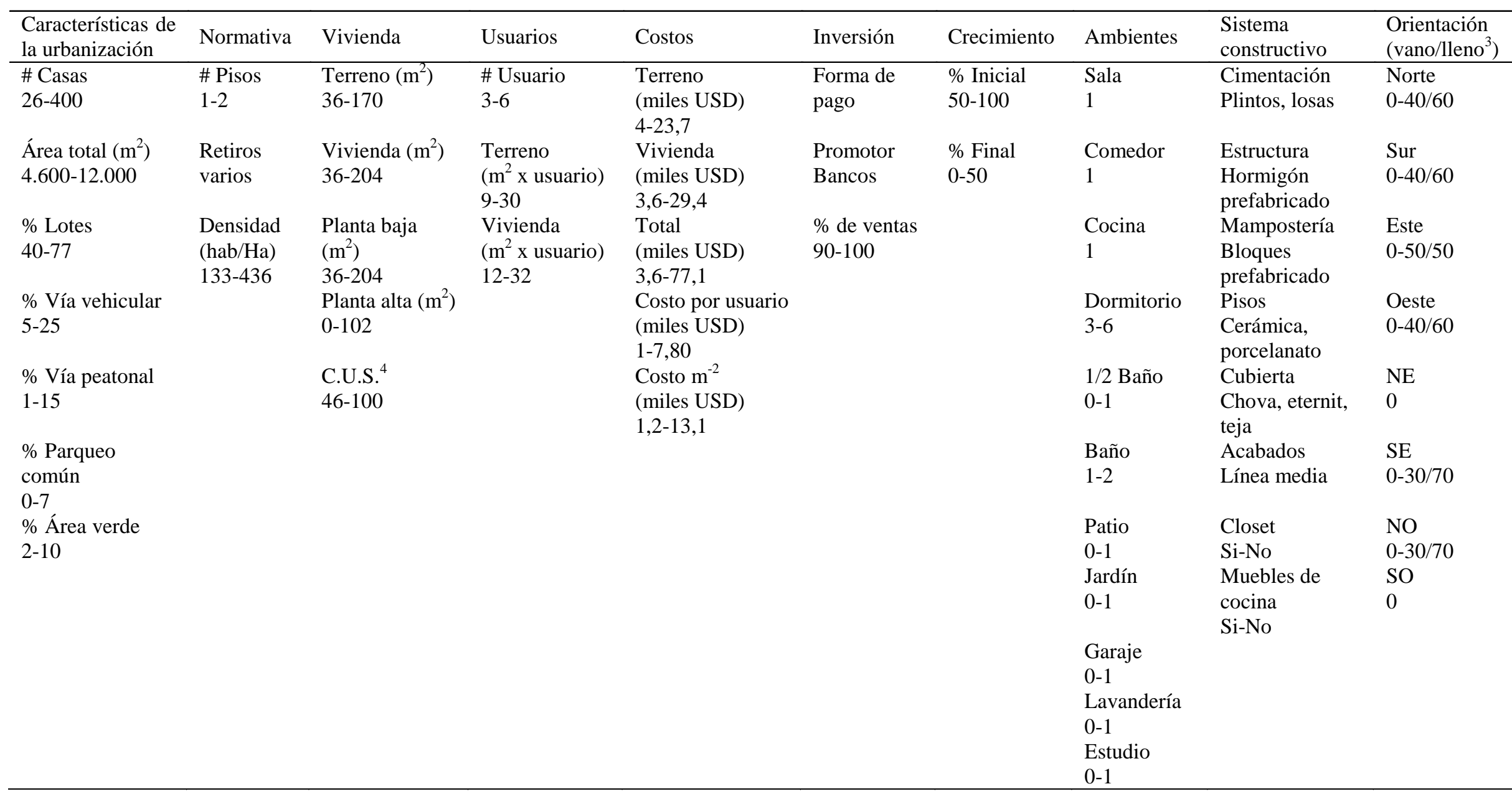

${ }^{3}$ Es la relación vano y lleno del volumen. Se entiende como vano las cavidades "huecos", mientras que el lleno es el volumen real.

${ }^{4}$ El coeficiente de uso del suelo, define la máxima superficie total de un predio.

Revista semestral de la DIUC 


\section{PROCESO DE DISEÑO}

\subsection{Aspectos del diseño de la vivienda}

Considerando los datos de la Matriz de Valoración (Tabla 1), se establecieron áreas y espacios para la planificación arquitectónica de la vivienda. Así también, se condicionó el diseño a lo establecido por la Ordenanza que Sanciona el Plan de Ordenamiento Territorial del Cantón Cuenca (1998), en donde se describen las áreas mínimas de los espacios de las edificaciones. La Tabla 2, muestra las áreas en función de las cuales se desarrolla el proyecto. La vivienda en una primera etapa tiene $75,42 \mathrm{~m}^{2}$, de construcción, a más de $36,14 \mathrm{~m}^{2}$ de áreas no computables (áreas verdes, lavandería, patio y garaje) que se implantan sobre un terreno de $81 \mathrm{~m}^{2}$. A diferencia de los planes analizados, la vivienda tiene la posibilidad de crecer en una segunda etapa en $16,52 \mathrm{~m}^{2}$, para albergar dos dormitorios adicionales, con lo que el área de construcción se incrementaría $91,94 \mathrm{~m}^{2}$, sin que repercuta en el confort lumínico o térmico.

Tabla 2. Áreas y planificación de la vivienda.

\begin{tabular}{llcc}
\hline Nivel & Espacio & $\begin{array}{c}\text { Área de diseño } \\
\left(\mathrm{m}^{2}\right)\end{array}$ & $\begin{array}{c}\text { Área según norma } \\
\left(\mathrm{m}^{2}\right)\end{array}$ \\
\hline \multirow{4}{*}{ Planta baja } & Sala-comedor & 10,02 & $\geq 7,30$ \\
& Cocina & 8,70 & $\geq 4,50$ \\
& Dormitorio (\# 1) & 10,58 & $\geq 7,29$ \\
& Baño social & 3,52 & $\geq 2,50$ \\
& Circulación & 11,98 & --- \\
& Total & 44,80 & $\geq 8,50$ \\
\multirow{5}{*}{ Planta alta } & Dormitorio máster (\# 2) & 10,58 & $\geq 7,29$ \\
& Dormitorio (\# 3) & 8,40 & $\geq 2,50$ \\
& Baño & 3,64 & $\geq 7,30$ \\
\hline Área computable & Vestíbulo & 7,40 & \\
\hline \multirow{4}{*}{ Área no } & Total & 30,62 & $\geq 2,25$ \\
computable (sin & Área verde & 75,42 & $\geq 3,00$ \\
construcción) & Lavandería & 4,6 & $\geq 7,5$ \\
& Patio posterior & 4,25 & \\
\hline Área computable & Garaje & 17,53 & \\
\hline & Total & 9,76 & \\
& Total & 36,14 & \\
\hline
\end{tabular}

Forma y orientación de la vivienda

En el terreno de $81 \mathrm{~m}^{2}$ (terreno rectangular de $11,25 \mathrm{~m} \mathrm{x} \mathrm{7,2} \mathrm{m),} \mathrm{las} \mathrm{fachadas} \mathrm{están} \mathrm{dispuestas} \mathrm{de} \mathrm{tal}$ forma que se asegura la iluminación y ventilación natural. Para conseguir esto, la vivienda se emplaza en su eje longitudinal en la dirección este-oeste, de tal forma que se logre la máxima radiación solar sobre sus fachadas. De esta manera, al utilizar técnicas pasivas, se espera disminuir el consumo energético (GhaffarianHoseini y col., 2013). En la Fig. 2, se distingue un bloque de casas, y los alzados frontal y posterior son las únicas fachadas que reciben la radiación durante las 12 horas de soleamiento; de esta forma se logra una penetración solar total hacia la vivienda, mejorando las condiciones de iluminación natural y las condiciones térmicas interiores. Además, tiene captadores

\footnotetext{
${ }^{5}$ Coeficiente de ocupación de suelo, es el máximo porcentaje construible de desplante en relación a la superficie total de un predio.
} 


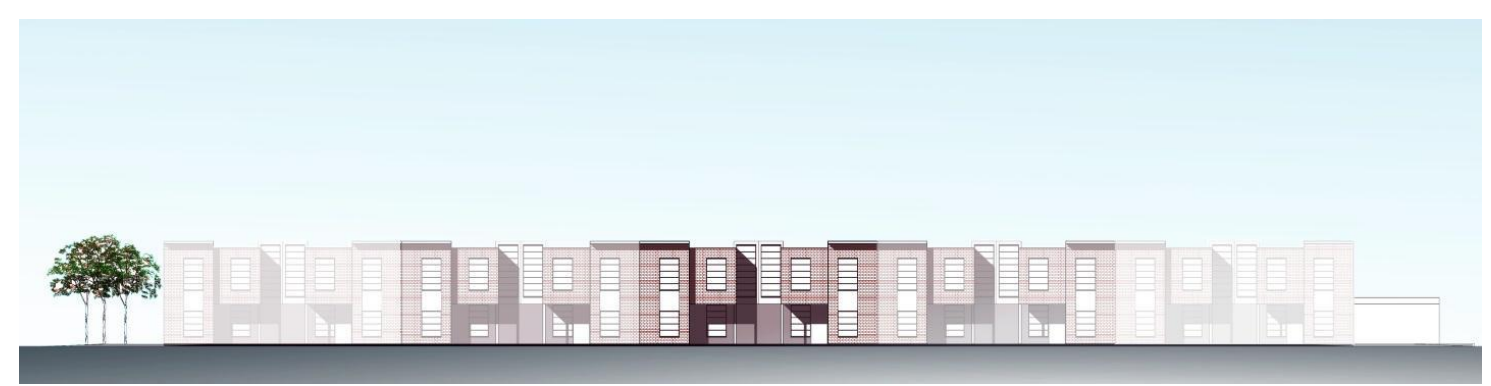

Crecimiento en el eje norte-sur

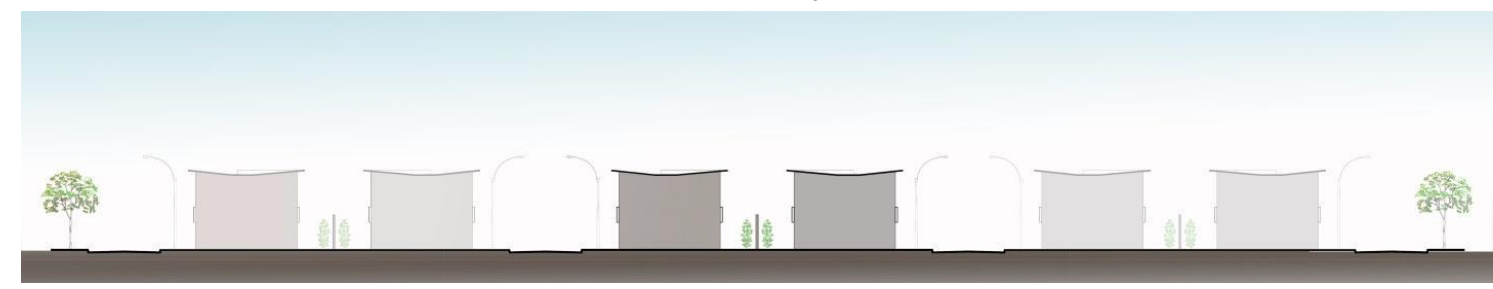

Crecimiento en el eje este-oeste
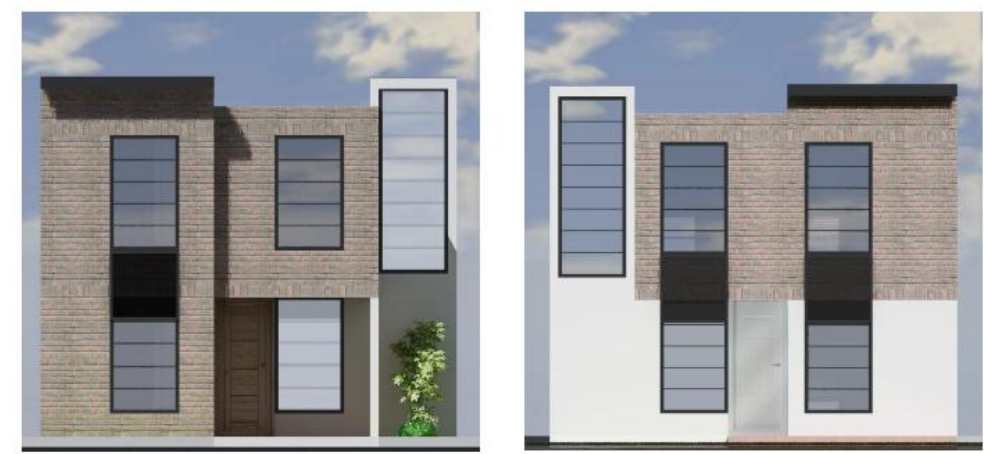

Alzado frontal - Este / Oeste (Izq.) y alzado posterior - Este/Oeste

Figura 2. Orientación de una fila de casas.

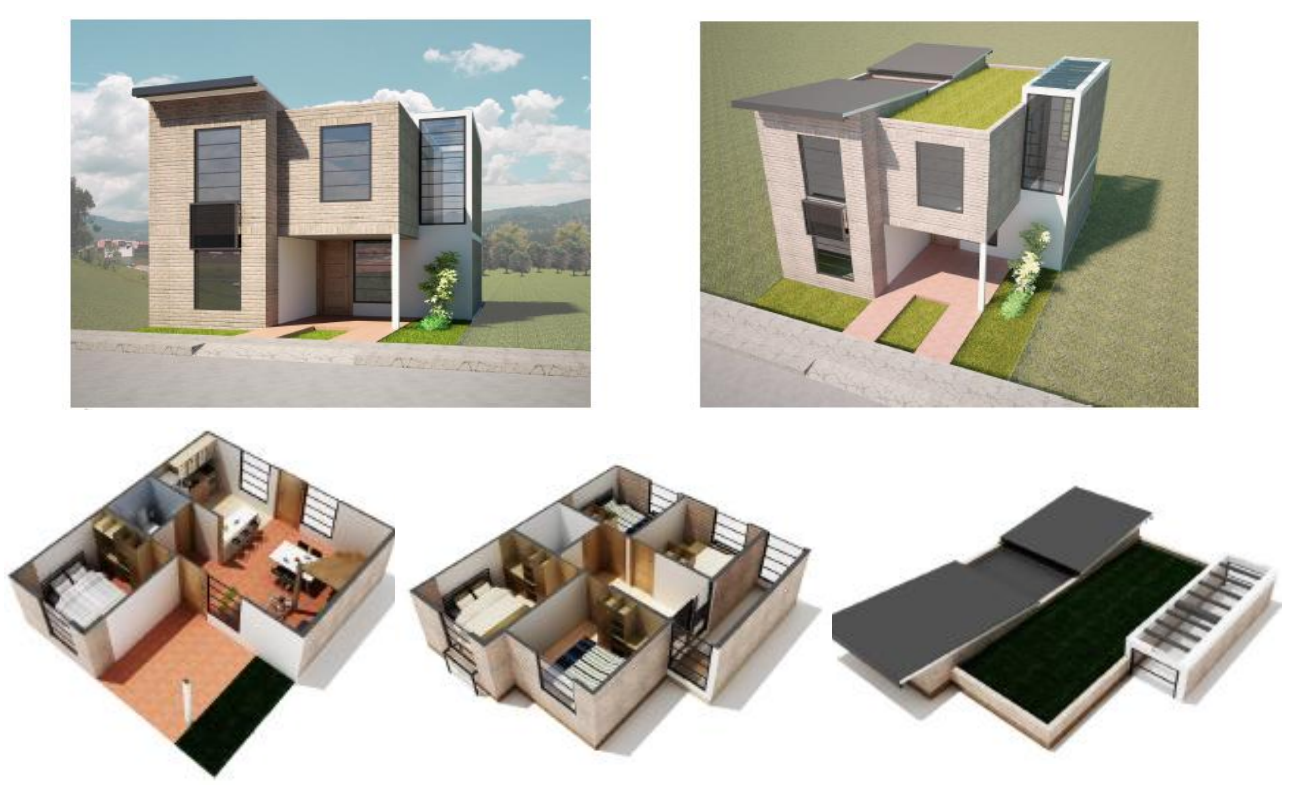

Figura 3. Vista de la unidad habitacional (parte superior de la figura), planta baja, segunda planta y techo (parte inferior de la figura, respectivamente, a la izquierda, central y derecha). 
en sus fachadas y cubierta. En las fechas próximas a los equinoccios del 21 de marzo y 21 de septiembre, el rendimiento bioclimático llega a $100 \%$, mientras que para las fechas próximas a los solsticios del 21 de junio y 21 de diciembre el rendimiento baja al $90 \%$, sin dejar de ser altamente eficiente.

\section{Conceptualización de la vivienda}

El concepto se basó en que la vivienda permita el aprovechamiento máximo de la radiación e iluminación solar, tanto al interior como exterior, así como también permita la ampliación de dos dormitorios, sin que se altere estas características. La Fig. 3, muestra las características de los espacios de la vivienda. Dentro de las características de los espacios de la vivienda se encuentra, en la planta baja: áreas verdes exteriores y posteriores, un garaje cubierto para un auto, zona de sala, comedor y cocina en un solo ambiente, dos baños completos, un dormitorio y estudio. Se advierte que la disposición es hacia la fachada frontal, con lo cual se garantiza al menos el acceso de una persona con capacidades diferentes a todos los espacios vitales. La zona de escaleras, también permite distribuir las ganancias térmicas hacia el interior de la vivienda. En la cubierta se dispone de sistemas de recolección de agua lluvia para reciclaje de este recurso y las cubiertas con las inclinaciones necesarias para la instalación posterior de paneles térmicos y/o fotovoltaicos.

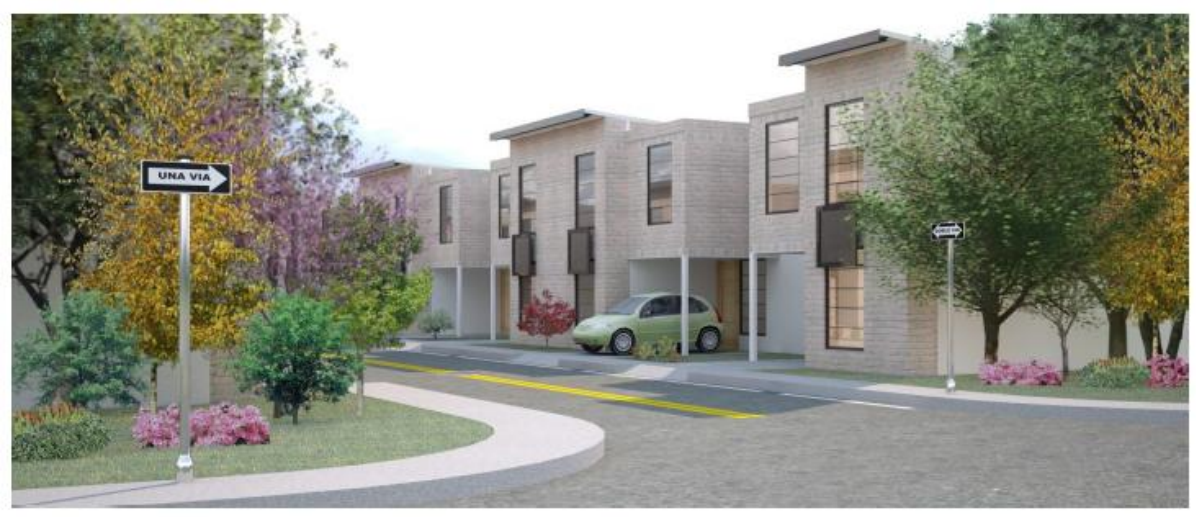

Figura 4. Esquema de distribución de la vivienda y expansión como urbanización.

La casa está concebida de tal manera que pueda ampliarse a dos dormitorios adicionales, sin que esto, implique el cambio de la fachada frontal de la etapa inicial de la construcción. Adicionalmente, el proyecto concibe a la vivienda no como una unidad habitacional, sino que permite la expansión horizontal, sin que esto ocasione que se pierdan las características de confort (Fig. 4). Esto da la pauta para que un proyecto de pequeña escala pueda ser extendido a una producción masiva (Seyfang, 2010), con la consecuente disminución de costos y a la vez promueva una difusión de la tecnología no solo a nivel profesional sino de usuario. El diseño de la vivienda asume además un enfoque holístico, adecuado para los países en vías de desarrollo, pues a la vez que provee calidad de vida a los usuarios, al mismo tiempo cubre una necesidad social y minimiza el impacto ambiental (Wekesa, 2010).

\subsection{Materiales}

Los tipos de materiales elegidos para una construcción, son determinantes que inciden en términos del impacto ambiental (Basbagill y col., 2013). Al no realizarse un análisis estricto del ciclo de vida de los materiales, para este estudio se planteó una preselección, en base a criterios de existencia en el medio (madera, ladrillo panelón, hormigón prefabricado, ladrillo de obra) y el uso de materiales que se consideran como menos contaminantes. Para establecer las características de los materiales en relación con el ambiente, se pueden considerar varios índices, como consumo energético en su fabricación, emisiones de $\mathrm{CO}_{2}$ (Cerón y col., 2013; GhaffarianHoseini y col., 2013), posibilidades de reciclaje o su comportamiento en relación a los atributos bioclimáticos referentes al confort, ya sea lumínico o térmico. Según Zabalza y col. (2011), el uso de madera, hormigón, concreto o ladrillos, reduce las 
emisiones de $\mathrm{CO}_{2}$. Además, el uso de materiales locales, evita el impacto asociado a su transporte (Zander y Sandström, 2012; Cabeza y col., 2013). Por ello, se optó por materiales que se los puede obtener con proveedores locales, con lo que se dispone de elementos más eficientes al clima, así como se disminuye la huella ecológica al disminuir su transporte (Cerón y col., 2013). Es decir, se genera una arquitectura con identidad de lugar, reactivando por tanto la economía local.

En el caso del impacto ambiental, se estableció que de los materiales utilizados, la chova (empleada para la cubierta), el acero (empleado para la estructura), o el hormigón (cimientos) tienen el mayor índice de gasto energético para su fabricación por kilogramo de material $\left(30 \mathrm{kWh} \mathrm{kg}^{-1}, 8,96\right.$ $\mathrm{kWh} \mathrm{kg}^{-1}$ y $2,82 \mathrm{kWh} \mathrm{kg}^{-1}$ respectivamente), mientras que materiales como el ladrillo (paredes), madera (carpintería, tabiquería), tienen índices energéticos bajos $\left(0,7 \mathrm{kWh} \mathrm{kg}^{-1}, 0,56 \mathrm{kWh} \mathrm{kg}^{-1}\right)$. En cuanto a las emisiones de $\mathrm{CO}_{2}$ por kilogramo de material relacionadas al proceso de fabricación, la chova, acero, hormigón, ladrillo y madera, generan respectivamente $1,78 \mathrm{~kg} \mathrm{CO} \mathrm{kg}^{-1}, 1,03 \mathrm{~kg} \mathrm{CO}$ $\mathrm{kg}^{-1}, 0,12 \mathrm{~kg} \mathrm{CO}_{2} \mathrm{~kg}^{-1}, 0,04 \mathrm{~kg} \mathrm{CO}_{2} \mathrm{~kg}^{-1}, 0,29 \mathrm{~kg} \mathrm{CO}^{2} \mathrm{~kg}^{-1}$ (Basbagill y col., 2013). En otras palabras, el uso de materiales elaborados industrialmente, incrementará la energía incorporada en la vivienda, así como las emisiones de $\mathrm{CO}_{2}$ asociadas.

De la validación realizada, se determinó que los materiales utilizados para la construcción permiten mantener el confort térmico, pues su capacidad de aislamiento o baja conductividad térmica posibilitan que la temperatura en el interior de la vivienda se mantenga dentro de las condiciones requeridas.

\subsection{Tecnologías limpias}

Agua

La vivienda dispone de un sistema de reciclaje de aguas lluvias, permitiendo que el líquido se almacene en contenedores ubicados en el ducto lateral, para permitir su uso en aparatos sanitarios, riego y limpieza, tal como propone GhaffarianHoseini y col. (2013). El diseño del techo, en este sentido, resulta esencial, pues permite el manejo sostenible del agua de lluvia, esto por cuanto, los techos representan aproximadamente la mitad de la superficie expuesta a la lluvia en las ciudades (Ramón, y col., 2011). La captación, recolección y distribución de agua de lluvia es un medio elemental de obtención de agua para uso humano específico y/o uso agrícola. En diferentes latitudes con mediana precipitación y en donde no se dispone de agua en cantidad y calidad mínima para consumo humano, se recurre al agua de lluvia como fuente de abastecimiento primaria. En el caso del proyecto, el agua de lluvia es captada, colectada y almacenada en depósitos para su posterior uso. En la captación del agua de lluvia para uso doméstico se emplea el modelo SCAPT (sistema de captación de agua pluvial en techos).

La producción u "oferta" de agua, está relacionada directamente con la precipitación durante el año y con las variaciones estacionales de la misma, mientras que la demanda depende de las necesidades del interesado, ya sea aparatos sanitarios, limpieza, riego, entre otros. Para dimensionar el sistema de almacenamiento de agua se consideró un promedio de captación de 20 litros diarios por familia. Esto se considera como adecuado para satisfacer las necesidades básicas de aparatos sanitarios y limpieza. El sistema de captación de agua de lluvia, consta de elementos de captación, recolección, conducción, almacenamiento y distribución. El sistema de almacenamiento está formado por seis tubos de $110 \mathrm{~mm}$ de diámetro, que en total pueden almacenar 480 litros de agua. El consumo diario se supone de 176 litros (10 para riego, 22 limpieza y 144 sanitarios). Puesto que la vivienda dispone de $56,25 \mathrm{~m}^{2}$ de superficie en el techo, la cantidad de agua que podría recoger es de 136,88 litros, si se considera una precipitación promedio de $73 \mathrm{ml} \mathrm{m}^{-2}$ por mes. Además, si se incluye el coeficiente de escorrentía, que depende del material del techo e inclinación ( 0,8 superficies planas y 0,9 superficies inclinadas), la cantidad que se podría almacenar considerando que el $34,84 \%$ de la superficie es plana, es de 118,42 litros de agua (Ramón, y col., 2011).

\section{Energía}

En Martínez (2010) se determina que los usos finales de la energía (electricidad y gas licuado de petróleo), en la ciudad de Cuenca, representan entre el 12 al 19\% de iluminación y entre 2 a 3\% de 
calentamiento de agua. Además, se indica que aunque el consumo de electricidad con fines de iluminación es más notorio entre las $18 \mathrm{~h} 00$ a $24 \mathrm{~h} 00$, en el día se presenta un uso de iluminación artificial, con picos entre las $6 \mathrm{~h} 00$ a $8 \mathrm{~h} 00$ y a las $14 \mathrm{~h} 00$.

Con el fin de garantizar la disminución de consumo energético, en el diseño de la vivienda se consideraron técnicas pasivas de control energético. Así por ejemplo, las fachadas (frontal y posterior) disponen de un sistema de muro trombe ${ }^{6}$, que calienta el aire desde la planta baja y la transmite hacia los dormitorios y a los materiales del entrepiso. La caja de gradas lateral funciona como un gran captador, generando un efecto invernadero que permite acumular el calor en las paredes de ladrillo laterales y en las escaleras, de esta forma, estos materiales puedan equilibrar la temperatura especialmente cuando ésta disminuye (noche). Todos los dormitorios están orientados hacia la salida y caída del sol, con lo cual se garantiza buenos niveles de iluminación natural directa y 6 horas de captación de sus fachadas. El pasillo interior de la segunda planta dispone de una puerta hacia la caja de gradas, con lo cual se evita pérdidas de calor una vez que se ha acumulado calor desde el invernadero lateral. Las paredes interiores son levantadas con paneles prefabricados de alta eficiencia en aislamiento acústico y térmico.

Las zonas centrales de mayor penumbra (pasillo y baño) disponen de iluminación cenital, evitando así la necesidad de consumo para iluminación. El diseño del cielo raso de la planta alta elaborado con paneles de madera, evita pérdidas de calor hacia la superficie fría de la cubierta. La losa central prevé su recubrimiento con capa vegetal, permitiendo así disponer de un elemento de excelente aislamiento térmico, y que a su vez permite recuperar capa vegetal al predio intervenido. La abertura de los aleros y la inclinación de la pendiente hacia el interior de la vivienda permiten mayor cantidad de tiempo para captación radiación solar sobre las fachadas. La cubierta traslucida sobre las escaleras garantiza 12 horas de sol al interior del invernadero, permitiendo así total ganancia en iluminación natural y ganancia térmica.

Según MEER (2008) para el 2020 se prevé un incremento en el uso de paneles solares térmicos con fines de calentamiento de agua caliente sanitaria (se espera un incremento de un 1\%), y una reducción del uso de gas licuado de petróleo utilizado para estos servicios. Considerando esto, se ha dimensionado la propuesta habitacional, de forma que la orientación e inclinación de las cubiertas sea adecuada para la instalación de paneles térmicos (para las latitudes de $2^{\circ}$, se aceptan inclinaciones de $10^{\circ}$ ), ya que éstos se encuentran ubicados en el eje este-oeste y no se provocan sombras entre sí. El dimensionamiento del sistema solar, consideró un consumo diario de 401 por usuario y una temperatura calórica de $50^{\circ} \mathrm{C}$. Como resultado se necesita, una superficie captadora de $2,54 \mathrm{~m}^{2}$, un tanque de almacenamiento de 200 litros, y un sistema auxiliar, que abastecería en un $21,90 \%$, de la energía que no suministraría el sistema solar térmico, en caso de que las condiciones climatológicas no sean apropiadas para su funcionamiento.

\subsection{Estimación del costo de construcción}

Para elaborar los costos de la construcción, se establecieron los rubros necesarios para la edificación: obras preliminares, cimentación de hormigón, acero de refuerzo, estructura de madera, encofrado, paredes, pisos, acabados, cubierta, puertas, carpintería metálica-ventanas, instalaciones sanitarias, instalaciones de agua potable, instalación eléctrica, muro trombe, escalera. El costo total, sin considerar terreno y el sistema térmico de agua fue de 16.019 USD, que representa 212,39 USD m². El rubro más representativo fueron los costos asociados a las paredes (18\%), seguidos de la estructura de madera, carpintería metálica ventanas (con 9\% cada una), hormigón (8\%), acabados e instalación eléctrica (7\%), puertas (6\%). El resto tiene valores porcentuales inferiores al $5 \%$.

El costo del terreno puede variar dependiendo de la ubicación, acceso e infraestructura; sin embargo, para mantener las condiciones de carácter social se asume un costo de $40 \mathrm{USD} \mathrm{m}^{-2}$ (precios en zonas rurales). Puesto que se requieren $81 \mathrm{~m}^{2}$, el costo total será de 19.259 USD. Si el costo del terreno se agrega a los valores referenciales anteriores, en este caso los porcentajes varían y el terreno significaría el $17 \%$ del costo total.

\footnotetext{
${ }^{6}$ Pared de mamposteria cubierta de una superficie acristalada. La radiación atraviesa el vidrio y calienta la masa permitiendo que el calor se acumule debido a que éste no puede atravesar el vidrio exterior.
} 


\section{VALIDACIÓN}

\subsection{El software Autodesk ECOTECT}

Autodesk ECOTECT Analysis es un software de análisis de diseño sostenible que ofrece una amplia gama de simulaciones y análisis de funcionamiento energético que permite mejorar el rendimiento de edificios existentes o el diseño de nuevas edificaciones. El software permite analizar condiciones de energía, uso de agua y emisiones de carbono, además posee herramientas que posibilitan visualizar y simular el comportamiento del edificio en el contexto de su medio ambiente. Con esta herramienta se realizó un análisis de la propuesta en relación con los parámetros térmicos y lumínicos. Para ello, se requirió información precisa sobre la localización del prototipo: latitud, longitud, altitud, temperatura, humedad, nivel de iluminación, entre otros.

Con la información meteorológica ingresada se pudo obtener simulaciones del comportamiento térmico y lumínico de la vivienda para diferentes épocas del año, y con diferentes situaciones climáticas. Los resultados del análisis térmico se determinaron para cada hora de los días correspondientes a los solsticios y equinoccios; es decir, para el día 21 de los meses de junio, diciembre, marzo y septiembre. Para ello, fue necesario identificar el tipo de material interior y exterior para evaluar cada ambiente según el número máximo de ocupantes.

El análisis lumínico consideró dos escenarios, el uno, con cielo despejado, y el otro, con cielo cubierto. El software, además permitió determinar las condiciones lumínicas para cada ambiente, es decir, analizó cada espacio considerando los colores de las paredes, iluminación natural o tamaño de las habitaciones.

\subsection{Validación de la propuesta}

En el caso del balance higrotérmico, se tuvieron en cuenta estrategias que están enmarcadas en el diagrama psicosométrico. Utilizando el software ECOTECT, se obtienen datos de temperatura interior durante las diferentes horas del día para cada espacio de la vivienda, para luego comprobarlos con la temperatura ambiente. Este software registra cada uno los espacios de la vivienda y los analiza térmicamente en base a la orientación, ubicación geográfica, forma y proporción, radiación directa, inercia térmica de los materiales, características de aislamiento, puentes térmicos, entre otros.

Se considera como fechas para el análisis térmico los solsticios ( 21 de junio y 21 de diciembre) y equinoccios (21 de marzo y 21 de septiembre), debido a que son las fechas en donde el sol llega a sus ángulos máximos de inclinación, a más de que la vivienda puede auto provocarse sombra cuando ésta se agrupe en un solo tramo de fachadas. Como resultado, el rendimiento térmico de los espacios interiores en las fechas analizadas, equinoccios del 21 de marzo y 21 de septiembre, y solsticios 21 de junio y 21 de diciembre, fue eficiente. Todos los espacios analizados (social y reposo) mantienen una constante de temperatura durante las 24 horas del día con promedios de $19,86^{\circ} \mathrm{C}$ en marzo, $20,27^{\circ} \mathrm{C}$ en junio, $19,92^{\circ} \mathrm{C}$ en septiembre y $20,59^{\circ} \mathrm{C}$ en diciembre, con un promedio anual de $20,16^{\circ} \mathrm{C}$. Estos resultados garantizan que la casa siempre cuente en sus espacios interiores con temperaturas de confort térmico, sin verse afectadas por las variables de amplitud térmica exterior. En algunas zonas, en horas del mediodía especialmente, el ambiente de la cocina se eleva por encima de los $24^{\circ} \mathrm{C}$, esto debido a la actividad que se realiza, pero con el sistema de ventilación cruzada puede eliminarse el calor innecesario y equilibrar las condiciones de confort.

Con el ECOTECT, además se valida los niveles de confort lumínico. Este programa calcula la incidencia solar directa en base a la posición del sol y tamaño y proporción de las ventanas, tomando en cuenta también la reflectividad de los materiales del interior. Se comprueba que todos los ambientes mantienen rangos de iluminación adecuados desde el panorama menos favorable que sucede cuando el cielo está nublado (iluminación exterior de 5000 lux aproximadamente). El método de medición aplicado consistió en tomar medidas en 4 puntos de cada ambiente a una altura de $70 \mathrm{~cm}$ para dormitorios y sala, y a $90 \mathrm{~cm}$ para la cocina. En caso de valores por encima del máximo establecido, se puede utilizar persianas o quiebrasoles internos, los cuales además no afectarían a las condiciones térmicas que dispone la vivienda. 


\section{DISCUSIÓN Y CONCLUSIÓN}

La metodología empleada vincula los elementos y factores del clima dentro del proceso de diseño de la vivienda, de esta forma el producto final constituye una respuesta integral. Se confronta la información del clima con el diagrama psicosométrico, lo que permite determinar la estrategia bioclimática más eficiente para la zona de estudio. Así mismo, empleando el diagrama de variación diaria de temperatura se determinan los rangos de confort térmico a lo largo del día para definir así las estrategias de acumulación y captación solar. De los cuatro índices, descritos en Malmqvist y Glaumann (2009), el estudio consideró el confort climático y visual, como los elementos que definen la satisfacción interior de los ocupantes. El sonido y la calidad de aire, al ser definidos por una ubicación específica, se los obvia; sin embargo, las estrategias pueden extenderse si se consideran estas variables.

A partir del análisis de los proyectos mencionados en la Matriz de Valoración, de los factores y elementos clima, se establecen las condiciones ambientales, y climáticas del área de estudio. A partir de la evaluación de los planes de vivienda mencionados, el proyecto plantea optimizar e intensificar dichos índices y criterios de diseño, considerando los valores funcionales, bioclimáticos, energéticos, sostenibles y económicos muchas veces omitidos. La información climática sirve para validar las condiciones de temperatura interiores, ya que son confrontadas con las temperaturas y humedades ambientales.

Todos los espacios de uso continuo (sala, comedor, cocina, estudio) se encuentran orientados al este y oeste, permitiendo la entrada de luz natural directa desde las $6 \mathrm{~h} 00$ hasta las 18h30. Los elementos estructurales y de mampostería han sido modulados dentro de los rangos de sus formas comerciales para evitar desperdicios y promover la construcción en seco de la vivienda en al menos un $90 \%$, con la consiguiente disminución de agua en la fase constructiva.

La estructura de columnas, vigas, entrepiso y cubierta es de sistema de armado en seco, empleando elementos en madera. Las puertas de acceso frontal y posterior están enfrentadas para favorecer la ventilación cruzada eficiente. La mampostería está resuelta de dos maneras: los muros de fachada de exposición directa con ladrillo panelón (material de muy baja energía incorporada) con lo cual se favorece los criterios de ganancia térmica de materiales, y los muros internos elaborados con paneles de hormigón prefabricados que debido a sus condiciones, tienen excelente comportamiento en aislamiento térmico y acústico, a más de que su materia prima es reciclable. Los pisos de la planta baja están recubiertos con ladrillo de obra, con lo cual se garantiza la captación térmica sobre su superficie y se evita pérdidas de calor hacia el suelo.

La vivienda, por la orientación de sus cubiertas, permite la colocación de energía solar, ya sea con paneles fotovoltaicos o fototérmicos. De la misma forma, considera la ubicación de acumuladores de agua lluvia para uso de sanitarios, riego u otras actividades de limpieza. El diseño modular, el sistema constructivo, materiales, cantidades y acabados, entre otros factores, determinan que el costo sea de 16.019 USD, con lo cual se está dentro del rango considerado para viviendas de interés social en el Ecuador. Con este presupuesto y con un costo referencial de 40 USD m ${ }^{-2}$ del terreno, se puede optar para el Programa del Bono que ofrece el Ministerio de Vivienda (20.000 USD) ${ }^{7}$.

Con el diseño del proyecto se contesta a la pregunta esencial de la investigación, es decir, es posible utilizar herramientas de diseño y técnicas de control medioambiental, incluso para diseñar viviendas de carácter social. Líneas de investigación, así como otras preguntas surgieron a lo largo del proyecto, entre ellas se anota: el análisis del ciclo de vida (Zander y Sandström, 2012), tanto en la etapa de construcción como operación (Cabeza y col., 2013), monitoreo de las características de confort y comportamiento de los usuarios (Peuportier y col., 2013), uso de energías renovables (especialmente la térmica, para agua de consumo sanitario y calefacción) (GhaffarianHoseini y col., 2013), el desarrollo de políticas para la promoción de eco viviendas (Zhang y col., 2011) y la comparación entre la calidad ambiental interior y el impacto ambiental (Assefa y col., 2010).

\footnotetext{
${ }^{7}$ Bono de la Vivienda: www.habitatyvivienda.gob.ec
} 


\section{AGRADECIMIENTO}

A la Dirección de Investigación de la Universidad de Cuenca, por la confianza entregada para realizar este trabajo. A los ayudantes de investigación: Juan Sarmiento, Vanessa Guillen, Andrea Gonzáles, por su paciencia e inagotables ganas. Al arquitecto Felipe Quesada, por su entrega en el inicio del Proyecto, y al Dr. Jan Feyen, por su desinteresada ayuda y guía.

\section{BIBLIOGRAFÍA}

Abdel, A., I. Aboulgheit, 2012. Assessing housing interior sustainability in a new Egyptian city. Procedia Soc. Behav. Sci., 564-577.

Assefa, G., M. Glaumann, T. Malmqvist, O. Eriksson, 2010. Quality versus impact: Comparing the environmental efficiency of building properties using the EcoEffect tool. Build. Environ., 45(5), 1095-1103.

Basbagill, J., F. Flager, M. Lepech, M. Fischer, 2013. Application of life-cycle assessment to early stage building design for reduced. Build. Environ., 60, 81-92.

Bodach, S., 2010. Energy in social housing: Opportunities and barriers from a case study in Brazil. Energ. Policy, 38(12), 7898-7910.

Cabeza, L., C. Barreneche, L. Miro, F.I. Martínez Mónica, D. Urge-Vorsatz, 2013. Affordable construction towards sustainable buildings: review on embodied energy in building materials. Curr. Opin. Env. Sust., 5(12), 229-236.

Cerón, I., E. Sanyé, J. Oliver, N. Motero, C. Ponce, J. Rieradevall, 2013. Towards a green sustaninable strategy for social neighbourhoods in Latin America: Case from social housing in Merida, Yucatan, Mexico. Habitat Int., 38, 47-56.

Edwards, B., 2013. Guía básica de la sostenibilidad (2ª edicion, $4^{\mathrm{a}}$ tirada). Editorial Gustavo Gilo, Barcelona, España, 224 pp.

Gonzalez, J.N., 2004. Arquitectura bioclimática. Editorial Munilla-Leira, Madrid, España, 443 pp.

Higueras, E., 2006. Urbanismo bioclimático (1ª Edición). Editorial GG, Barcelona, España, 242 pp.

GhaffarianHoseini, A.H., N.D. Dahlan, U. Berardi, A. GhaffarianHoseini, N. Makaremi, M. GhaffarianHoseini, 2013. Sustainable energy performances of green buildings: A review of current theories, implementations and challenges. Renew. Sust. Energ. Rev., 25, 1-17.

Larrea, C., 2010. La situación habitacional en Pichincha y en el Ecuador: 2001-2010. Unidad de Información Socio-ambiental, Universidad Andina Simón Bolívar sede Quito, Ecuador, 19 pp. Descargado de http://www.campusvirtual.uasb.edu.ec/uisa/images/publicaciones/ 2011_larrea_habpich.pdf en 2013.

Malmqvist, T., M. Glaumann, 2009. Environmental efficiency in residential buildings. A simplified communication approach. Build. Environ., 44(5), 937-947.

Martinez, P., 2010. Usos finales de energía eléctrica y GLP en el cantón Cuenca. Escenarios al año 2015. Tesis previa para obtener el Título de Master en Gestión Tecnológica, Universidad de Cuenca, Cuenca, Ecuador, 139 pp.

MEER, 2008. Matriz energética del Ecuador. Ministerio de Electricidad y Energías Renovables, Quito, Ecuador.

Murillo, R., 2011. Metodología para el diseño arquitectónico bioclimático en zonas de clima caliente y húmedo. Facultad de Arquitectura y Diseño, Universidad Católica de Santiago de Guayaquil, Guayaquil, Ecuador, 82-91. Descargado de http://www2.ucsg.edu.ec/dmdocuments/AUC-31.pdf. el 31 de agosto del 2013.

Neila, G., 2001. Arquitectura bioclimática en un entorno sostenible. Editorial Munilla-Leira, Madrid, España, 440 pp. 
Pacheco-Torga, F., S. Jalali, 2012. Earth construction: Lessons from the past for future eco-efficient construction. Constr. Build. Mater., 29, 512-519.

Paz Jácome, J.F., F.V. Ulloa López, 2006. La vivienda de interés social. Revista Ingenius, 3 , 8 pp.

Peuportier, B., S. Thiers, A. Guiavarch, 2013. Eco-design of buildings using thermal simulation and life cycle assessment. J. Clean Prod., 39, 73-78.

Ramón, F., Morales-Pinzón, T., Guisasola, A., Taya, C., Rieradevall, J., \& Gabarrell, X. 2011. Roof selection for rainwater harvesting: Quantity and quality assessments in Spain. Water Res., 45(10), 3245 - 3254.

Serra, R., 2002. Arquitectura y clima. Editorial Gustavo Gili S.A., Barcelona, España, 7-94.

Seyfang, G., 2010. Community action for sustainable housing: Building a low-carbon future. Energ. Policy, 38(12), 7624-7633.

Wekesa, B.S.G., 2010. The response of common building construction technologies to the urban poor and their environment. Buil. and Environ., 45(10), 2327-2335.

Zabalza, I., A. Valero, A. Aranda, 2011. Life cycle assessment of building materials: Comparative analysis of energy and environmental impacts and evaluation of the eco-efficiency improvement potential. Build. Environ., 46(5), 1133-1140.

Zander, J., R. Sandström, 2012. Materials selection with several sizing variables taking environmental impact into account. Mater. Des., 37, 243-250.

Zhang, X., L. Shen, Y. Wu, 2011. Green strategy for gaining competitive advantage in housing development: a China study. J. Clean Prod., 2(19), 157-167. 